\title{
KELEA: A Natural Energy That Seemingly Reduces Intermolecular Hydrogen Bonding in Water and Other Liquids
}

\author{
W. John Martin \\ Institute of Progressive Medicine, South Pasadena, CA, USA \\ Email: wjohnmartin@ccid.org
}

Received 8 June 2015; accepted 14 July 2015; published 17 July 2015

Copyright (C) 2015 by author and Scientific Research Publishing Inc.

This work is licensed under the Creative Commons Attribution International License (CC BY).

http://creativecommons.org/licenses/by/4.0/

(c) (i) Open Access

\begin{abstract}
The alternative cellular energy (ACE) pathway was initially identified as a non-immunological defense mechanism against virus infections. It is particularly relevant to the suppression of stealth adapted viruses, which are not normally recognized by the cellular immune system. Many of the methods able to enhance the ACE pathway are consistent with the transfer of a natural energy to the body's fluids. Additional support for this premise is provided in this paper. The vapor pressure and rate of evaporation of activated water, ethanol and gasoline increase to beyond atmospheric pressure over time. The term KELEA (kinetic energy limiting electrostatic attraction) is proposed for a natural energy that increases the volatility of fluids, seemingly through the loosening of intermolecular hydrogen bonding. KELEA activated fluids have many potential health, agricultural and industrial applications, as well as providing the opportunity for fundamental research.
\end{abstract}

\section{Keywords}

Alternative Cellular Energy, ACE Pathway, Magnesium Oxide, KELEA-Kinetic Energy Limiting Electrostatic Attraction, Vapor Pressure, Volatility, Stealth Adapted Viruses, Water, Alcohol, Gasoline, Kiko $^{\mathrm{TM}}$ Technology, Enercel $^{\mathrm{TM}}$, Homeopathy, Neutral Red Dye, Lidocaine

\section{Introduction}

The following is a brief synopsis of the events leading to the findings reported in this paper. Atypical viruses were detected in patients with neuropsychiatric illnesses using molecular based methods [1] [2]. The infections were subsequently confirmed using virus cultures [3]. Although sharing similar cell damaging, cytopathic effect (CPE) in cultures; the viruses were molecularly heterogeneous [4]. Moreover, the patients from whom the vi- 
ruses were cultured had a wide range of illnesses, sometimes differentially affecting various family members. Prominent illnesses among virus culture positive patients were; chronic fatigue syndrome, schizophrenia, bi-polar psychosis, childhood behavioral disorders and autism [5]-[8]. The lack of an inflammatory reaction in these diseases or in virus inoculated animals [9] led to the designation of stealth adapted viruses.

Damaged cells in the virus cultures will typically recover if the tissue culture fluid (medium) is not replaced. The cellular recovery is attributed to the accumulation of pigmented particles and fibers. These materials are fluorescent when illuminated with ultraviolet (UV) light, especially if mixed with certain dyes including acridine orange, stains-all and neutral red. The particles are also commonly electrostatic and occasionally ferromagnetic. Replacement of the tissue culture medium with fresh medium leads to the rapid reactivation of the CPE. The reactivation can be prevented by including some of the particles from repaired cultures in the re-feeding medium [10] [11].

The CPE from stealth adapted viruses had earlier been shown to be reduced by the addition of a supposedly homeopathic product called HANSI (homeopathic activator of the system immune) in the tissue culture medium. Based on the tissue culture finding, the US manufacturer of HANSI changed its name to Enercel ${ }^{\mathrm{TM}}$. Gas Chromatography-Mass Spectroscopy (GC-MS) analysis by the author showed that Enercel ${ }^{\mathrm{TM}}$ contained a detectable level of lidocaine (xylocaine). Enercel ${ }^{\mathrm{TM}}$ is an effective therapeutic in many clinical conditions including tropical diarrhea [12], amyotrophic lateral sclerosis [13] and tuberculosis [14].

Neutral red dye had been successfully used in the phototherapy of herpes simplex virus (HSV) induced skin lesions in the early 1970's [15]. The findings were largely discounted, however, following failed attempts at replication using incandescent lighting [16]. Dr. Jon Stoneburner was continuing to achieve success using UV lighting in conjunction with neutral red dye. The herpes lesions were noted to fluoresce during the phototherapy [17] [18]. Because of concern that neutral red dye could possibly lead to DNA alterations in the herpes virus genome, the procedure was modified. A lidocaine containing, ethanol-based herbal solution with freshly added neutral red dye was used to moisten paper towels laid onto the herpes skin lesions. Upon UV illumination, the solution applied to the paper towel fluoresced, but so too did the underlying skin lesion when it was directly illuminated with the UV light. Direct UV fluorescence could commonly also be seen in the uninvolved skin adjacent to the herpes lesion and, in the case of genital herpes, even within nearby public hairs. The indirectly treated herpes lesions underwent the same expedited recovery as when the neutral red dye was directly applied to the lesions [19].

The procedure was next adapted to allow parents to treat their own children with autism [20]. Remarkable clinical improvements were obtained when parents sprayed the lidocaine-containing solution onto paper towels, moistened with neutral red dye. The towels had been laid onto various Saran ${ }^{\mathrm{TM}}$ wrap covered skin areas of the children. This procedure readily evoked skin fluorescence both in the underlying skin and in patchy areas elsewhere in the child's body. While early treated patients had shown marked clinical improvements, often in spite of initial skepticism of the parents, later parents had the disappointment of seeing no benefits. The lidocainecontaining herbal solution being used had clearly lost its therapeutic activity.

The issue was investigated in a more limited study involving a parent willing to repeatedly treat her teenage daughter. Various methods were devised for activating ethanol-containing solutions, such that they would very strongly fluoresce with neutral red dye. Some of the methods simply involved the application of external energies, while other involved the addition of various components, with or without lidocaine. Instead of using paper towels, the activated solution was placed into Ziploc ${ }^{\mathrm{TM}}$ bags. A small quantity $(\sim 0.1 \mathrm{mg} / \mathrm{ml})$ of neutral red dye was added to the solution within the bag. The closed bag was typically placed against the sole of one or both feet and illuminated for approximately 30 minutes. In addition to clinical improvement, direct UV fluorescence would elicit bright fluorescence in other areas of the child's body including the tongue [20]. In some studies, the portion of the bag containing the solution was heat-sealed to avoid leakage.

The purpose of this article is to i) illustrate the increasing vapor pressure of activated solutions in heat-sealed flexible containers; ii) to explain the loss of activity in the solution being used in the earlier study; iii) describe a natural force tentatively called KELEA; and iv) outline some of the many applications of KELEA activated fluids.

\section{Material and Methods}

Neutral red dye (CAS 553-24-2) was purchased from Dudley Corporation, Lakewood NJ. Lidocaine (catalog No. L7757); magnesium oxide (MgO, catalog No. 220361) and absolute ethanol (catalog No. E7023) were pur- 
chased from Sigma-Aldrich, Saint Louis MO. Everclear (75.5\% alcohol), Stroh's Rum (80\% alcohol) and other alcoholic beverages were purchased from BrevMo liquor store, Pasadena CA. Arrowhead distilled water was purchased from Safeway. Capped 1.25 ounce (oz) glass vials and capped polypropylene $50 \mathrm{ml}$ bottles were obtained from The Container Store, Pasadena CA.

A Sartorious Digital Balance, measuring to $0.1 \mathrm{mg}$ was used for most measurements. A small Van de Graaff generator model C6917 purchased from Chaney Electronics Inc. Scottsdale AZ was used to activate condensing steam and ethanol vapor. Heating of the water or ethanol was achieved using a Haake Buchler MR 2002 heater with piping from a kettle to a heat resistant plastic bag that enclosed the dome of the Van de Graaff generator. This arrangement allowed for the collection of the condensate. Zero Technologies LLC, Bensalem PA, manufactured the Zero Water ion exchange filters used. Ziploc ${ }^{\mathrm{TM}}$ bags were heat sealed using a Seal-N-Save press from Sears. An ophthalmic tonometer with an upper limit of $30 \mathrm{mmHg}$ was used on one occasion.

\section{Results}

Ethanol solutions of neutral red dye were contained in heat-sealed portions of Ziploc ${ }^{\mathrm{TM}}$ bags. The containers were retained following their preparation for possible experimental/clinical use. Although the containers were initially flaccid, they expanded over the ensuring months. The expansion was generally first noticeable at about one month and gradually increased over the next 2 - 3 months. Indeed, some of the containers actually burst spilling their contents. Those that did not burst have stayed expanded for over 3 years with gradual reduction in the volumes of the contained fluid. Figure 1 shows the marked expansion of section of a Ziploc ${ }^{\mathrm{TM}}$ bag, which had been thermally sealed after the addition of a solution of neutral red dissolved in activated ethanol. The containers remain expanded even after all of the fluid had been lost, although the pressure then begins to slowly decrease over time; but not back to the initial flaccid state. Figure 2 shows an expanded Ziploc ${ }^{\mathrm{TM}}$ bag heat-sealed just below its zip lock closure. The bag originally contained approximately $30 \mathrm{ml}$ of activated ethanol plus neutral red dye. The volume of fluid in the expanded bag gradually decreased over time, until the Ziploc ${ }^{\mathrm{TM}}$ bag was dry. Nevertheless, the bag has remained expanded for over 2 years.

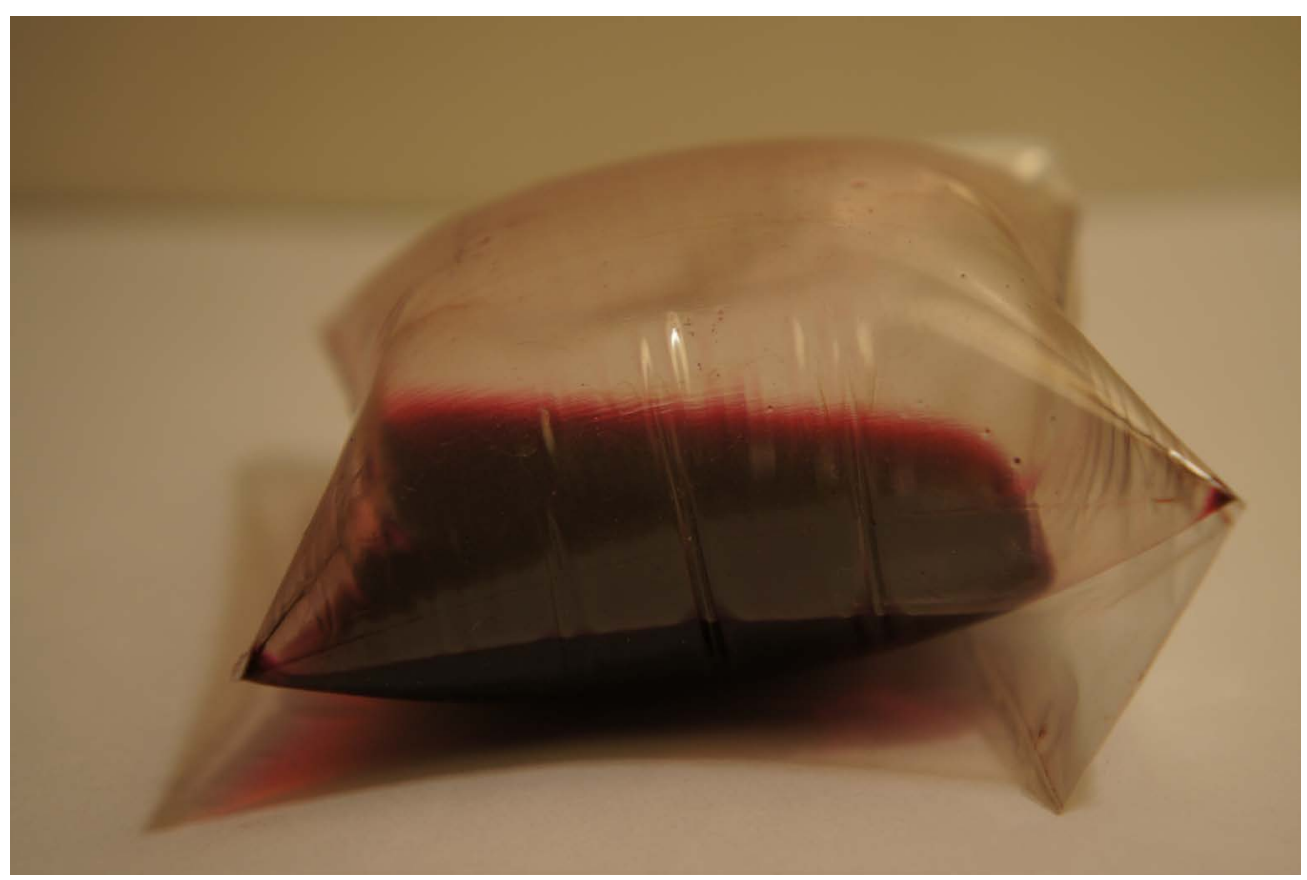

Figure 1. A photograph showing the marked expansion and stretching of a thermally sealed portion of a Ziploc ${ }^{\mathrm{TM}}$ bag, into which a solution of activated ethanol plus neutral red dye had been added before sealing. The ethanol had previously been activated using a combination of herbal products plus lidocaine. The bag was initially flaccid, but became progressively expanded over the ensuring weeks to several months. This particular container has remained expanded for over 2 years with a noticeable slow decrease in the residual fluid level. 


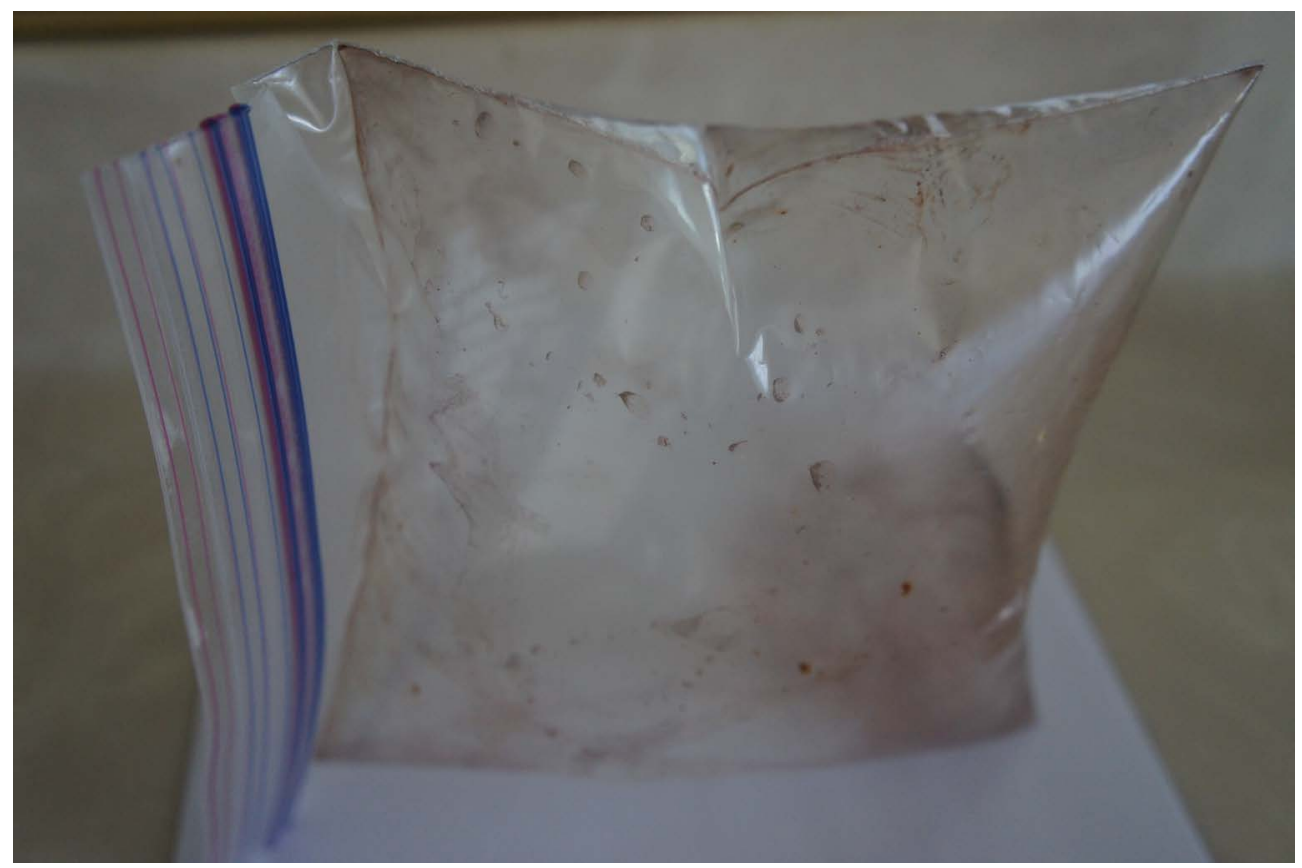

Figure 2. Photograph of an expanded Ziploc ${ }^{\mathrm{TM}}$ bag. Approximately $30 \mathrm{ml}$ of activated ethanol plus neutral red dye had previously been added to the bag, which was then heat sealed just below the interlocking closure. The bag underwent considerable expansion while retaining much of the original solution. The amount of fluid slowly diminished over approximately a year, leaving a still expanded bag with no residual fluid. The fluid-less bag has remained expanded for more than 2 years, with a gradual reduction in volume.

Tests were performed to determine whether the neutral red dye or other components in the alcohol was playing a critical role in the expansion. Absolute ethanol was heated in a kettle and its vapor was condensed over the dome of a Van de Graaff generator. The ethanol was collected and approximately $30 \mathrm{ml}$ aliquots were placed into Ziploc ${ }^{\mathrm{TM}}$ bags. The regions of each bag containing the fluid were heat-sealed resulting in flaccid containers. The containers were left at room temperature along with similar containers of non-activated ethanol. The containers with the activated ethanol became progressively expanded over the next several months. Placing the tightly expanded containers into a refrigerator decreases the pressure but not back to becoming flaccid. Furthermore, the pressure promptly increased to its earlier value upon removing the containers from the refrigerator. Figure 3 shows an expanded container of ethanol approximately 2 years after it initially became expanded. Using an ophthalmic tonometer the pressure in the expanded container exceeded $30 \mathrm{~mm} \mathrm{Hg}$ (the upper level of recording). No expansion over time has occurred in sealed containers with non-activated ethanol.

Rather than using heat-sealed plastic containers, a series of experiments were performed in glass vials and polypropylene bottles that were tightly capped after near complete filling with various fluids. The vials and bottles were then periodically weighed to determine the rate and extent of weight loss over time.

Again a very clear distinction was seen between the minimal weight loss of non-activated ethanol (generally $<0.1 \mathrm{mg} / \mathrm{ml}$ over 4 hours) and that of activated ethanol (invariably $>0.5 \mathrm{mg} / \mathrm{ml}$ and sometimes reaching several $\mathrm{mg} / \mathrm{ml}$ over 4 hours). The weight loss was prevented when the junction of the cap and the glass vial was completely sealed using putty and multiple layers of self-adhering silicone tape.

A similar assay using screw capped glass vials and plastic bottles was devised to distinguish between distilled water to which a small quantity of either activated or non-activated ethanol had been added. A 5-fold increase in the weight loss of distilled water was routinely achieved using as little as a 1:50 ratio of added activated ethanol to water when compared to the minimal, although still measurable effect of adding regular ethanol to water.

Various methods of ethanol activation have been evaluated using the weight loss assay and have also been applied directly to the activation of water. For example, water becomes activated as measured in the weight loss assay, by condensing steam over the dome of a running Van de Graaff generator. Also being placed close to a UV illuminated container of activated ethanol plus neutral red dye for several hours can lead to the water 


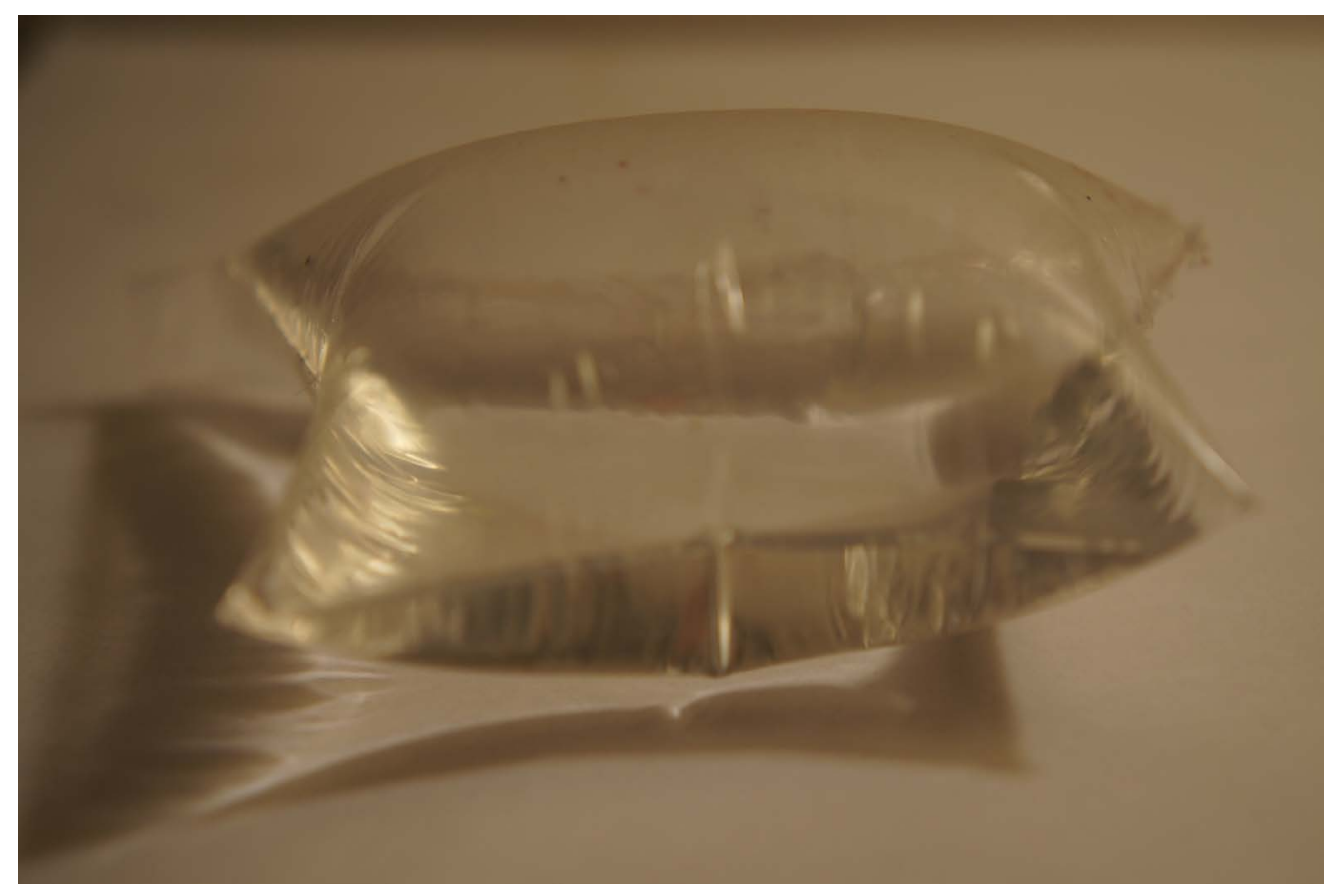

Figure 3. An expanded portion of a Ziploc ${ }^{\mathrm{TM}}$ bag that was heat-sealed after adding approximately 30 $\mathrm{ml}$ of activated ethanol. The ethanol had been activated by prior condensing ethanol vapor onto a dome of a running Van de Graaff generator. As in the example shown in Figure 1, the container was initially flaccid but by about a month began to expand. By 3 months, the container was markedly expanded and has remained so for over 2 years with gradually reducing amount of fluid.

becoming activated.

Various compounds added to water or to alcoholic beverages can lead to their activation, as measured by a > 5 -fold weight loss over time in filled, tightly capped glass vials. Moreover, once the water is activated, zero residue filtration can remove the activating compound without an appreciable early reduction in its ongoing rate of weight loss through vaporization. Many of the compounds used by organic farmers to enhance crop productions have been included in this testing. They include zeolites, humic/fulvic acids, volcanic rock pellets, mica, shungite (a product from Russia), and MgO granlues. Extracts of Moringa oleifera tree leaves and several pharmaceutical products in addition to lidocaine, have similarly been shown to have water and alcoholic beverage activating activities; using the accelerated weight loss assay (data not shown).

An experiment was performed with $\mathrm{MgO}$ to test for the duration of its ability to increase the vaporization of an alcoholic beverage called Stroh's Rum (80\% ethanol). A control vial with no added MgO and a test vial with 4 grams of MgO granules were filled with the rum and capped. The tops of both vials were wrapped with aluminum foil to help reduce ordinary evaporation. A second control vial had approximately 8 grams of $\mathrm{MgO}$ in addition to the rum. This cap on this vial was completely sealed using putty and multiple layers of self-adhesive tape. The 3 vials are shown in Figure 4. At 24 hours the weight of the control vial with no MgO had dropped by $8.0 \mathrm{mg}$, while the weight of the test vial had dropped by $47.6 \mathrm{mg}$. There was no weight loss in the completely sealed vial.

The test vial with added $\mathrm{MgO}$ granules and the first control vial were repeatedly weighed at relatively short time intervals over the first 40 days and then less frequently over the ensuring months. The cumulative weight losses for the two vials over the first 40 days, expressed as mg versus elapsed time in hours are shown in Figure 5. The figure shows continuing greater weight loss from the vial with the added MgO. There is also noticeable variation in the rate of weight loss over the time periods. The rate of weight loss of the vial with the added MgO gradually lowered over time but still remained significantly greater than that of the control vial even at the end of a year. The cumulative weight loss over the year in the test vial was 2.992 grams; compared to the year's loss of 0.523 grams in the control vial. Furthermore, the solution in the treated vial was colorless. While this was due in part to absorption of material by the $\mathrm{MgO}$ granules, it was likely also contributed to by a higher volatility of the 


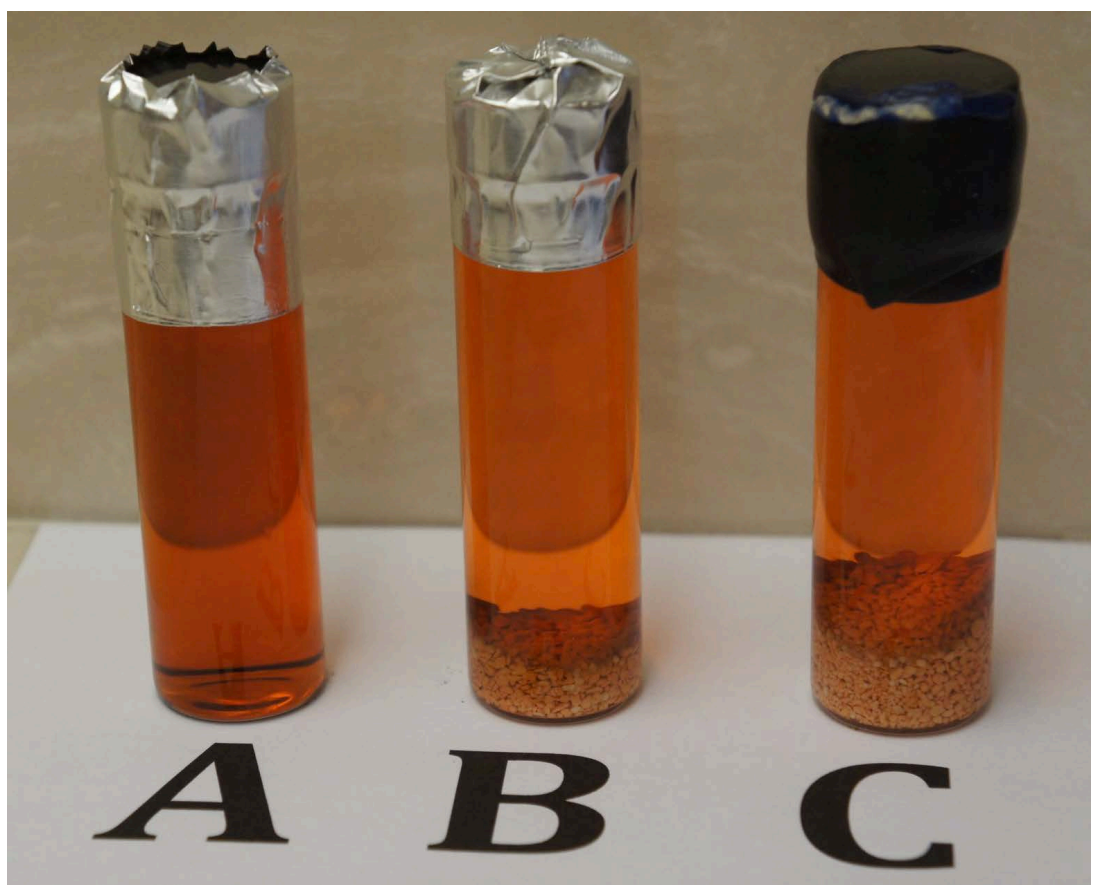

Figure 4. Three $1.25 \mathrm{oz}$ vials containing Stroh's rum. Vials B and C also contained approximately 4 gms and 8 gms of $\mathrm{MgO}$ granules, respectively. After tightly closing the screw top caps on all three vials, aluminum foil was wrapped around the caps of vials $\mathrm{A}$ and $\mathrm{B}$. For vial $\mathrm{C}$, ample putty was used to completely close the space between the lower edge of the cap and the glass bottle and to cover the rest of the cap. Multiple layers of a self-adhering silicone tape were then applied to the cap and top part of the bottle. This level of closure is required to prevent vaporization of activated ethanol and alcoholic beverages, such as Stroh's rum and Everclear.

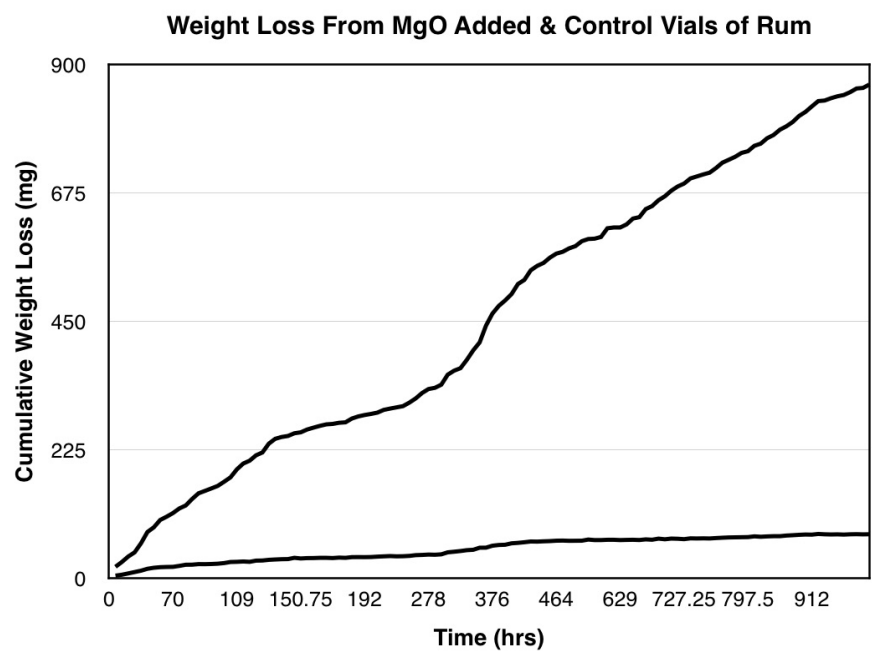

Figure 5. The cumulative losses of weight of the non-completely sealed vial of Stroh's rum with added MgO granules (top line) and that of the vial that had no added $\mathrm{MgO}$ (lower line). One hundred and twenty two different weighing's of each of the two vials were conducted at various times over the first 40 days. The times of weighing were recorded and expressed as hours plus 15-minute intervals, from the time of the initial readings. The figure shows the increasing greater weight loss of the vial containing $\mathrm{MgO}$ compared to the control vial. Slight differences in the interval rates of weight loss of the vial containing the $\mathrm{MgO}$ are apparent, with a definite slowing from approximately 120 to 220 hours. 
colored components in the rum when compared with the remaining solution. A photograph of the test and the first control sample at the end of the year is shown in Figure 6.

MgO has similarly been shown to increase the volatility of gasoline. Figure 7 shows the increased rate of weight loss in a $1.25 \mathrm{oz}$ capped glass vial of gasoline following the addition of 4 grams of MgO granules. This vial and a control vial had earlier shown equivalent weight losses of $\sim 2 \mathrm{mg} /$ hour. The results are plotted as weight loss in mg divided by the number of hours from the prior measurement. The data are plotted in this way to not only indicate that MgO increases the rate of weight loss but to further demonstrate the considerable variability in the rate of weight loss in the vial with added MgO. In similar experiments, the accelerated weight loss of gasoline exposed to $\mathrm{MgO}$ has been shown to continue for several days even after removal of the MgO.

\section{Discussion}

It is not uncommon for certain reported observations, to be disregarded by mainstream researchers because they are not easily explained within the standard belief systems. Moreover, active opposition can occur to new ideas that challenge existing economic or political interests.

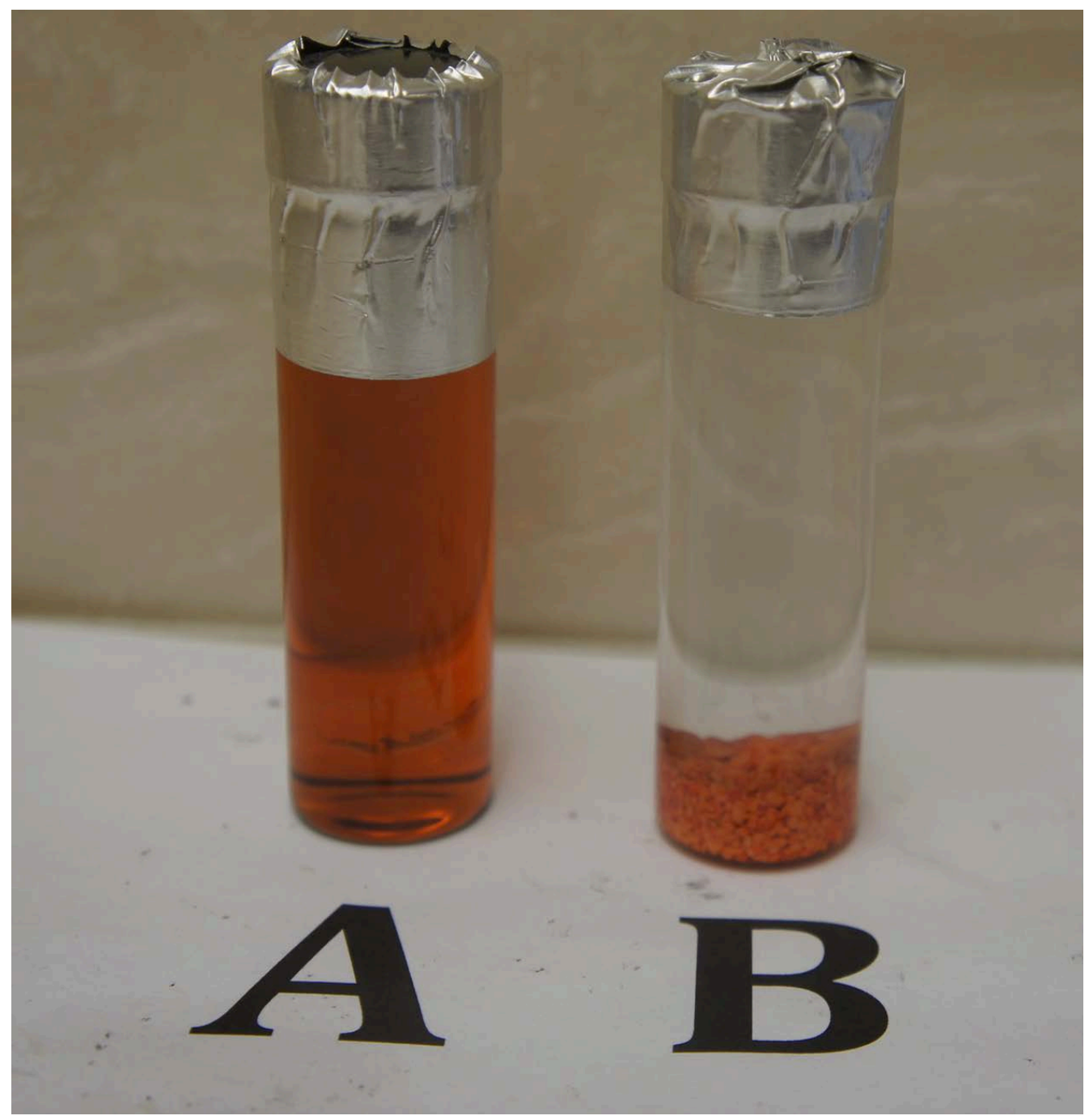

Figure 6. A photograph taken of the vial that had no added $\operatorname{MgO}(\mathrm{A})$ and the non-completely sealed vial of Stroh's rum with added $\mathrm{MgO}(\mathrm{B})$ one year after the beginning of the study. The solution in the $\mathrm{B}$ vial had been colorless for approximately 6 months. While the MgO had earlier absorbed some of the coloring in the rum, most of it had seemingly been more slowly lost through an increased rate of evaporation of the colored components compared to the remaining fluid. 


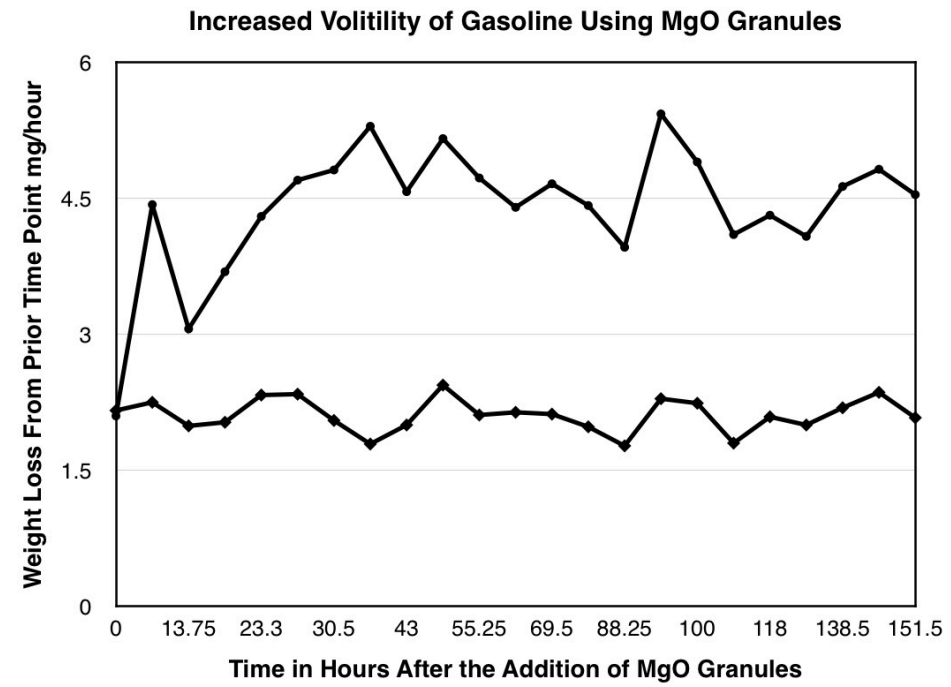

\begin{abstract}
Figure 7. A graph showing the rate of weight loss, expressed as the reduction in weight in milligrams (mg) divided by the time in hours from the prior measurement. The experiment compared two tightly capped vials of gasoline that were both previously losing weight at essentially the same $2 \mathrm{mg} /$ hour. Four grams of $\mathrm{MgO}$ granules were added to one of the vials that was again tightly re-capped. This vial showed a persistent increase in weight loss of approximately $4.5 \mathrm{mg} /$ hour (upper line). The data are expressed in this manner to demonstrate the variability that occurs in the precise rate of weight loss especially in the test sample
\end{abstract}

Farmers have historically recognized that water from different sources varies in the ability to support the cultivation of crops. Farmers have also shown benefits from adding various compounds in the water supply. The variable quality of water is generally attributed to differences in $\mathrm{pH}$ and/or mineral content, rather than possible alterations in an intrinsic "living" property of water.

Homeopathy [21] has also been met with skepticism partly due to the unsubstantiated claim that each formulation had to be specifically matched to the patient's symptom (Law of Similars). In reality, products such as Enercel $^{\mathrm{TM}}$, are broadly beneficial for many medical conditions [12]-[14] [22].

Oriental medicine champions the concept that body functions are partially regulated by life forces termed chi, prana, etc. [23]. Again, the lack of reliable detection methods for these forces has discouraged widespread acceptance.

The major new paradigm stemming from the research leading to the present study is that of an alternative cellular energy (ACE) pathway in humans, animals and plants. The ACE pathway seemingly involves an external energy force that can activate biological fluids. The focus of the present article is on direct evidence for an external force that increases the volatility and by inference the activation of various fluids. Future articles will provide data showing enhanced internal kinetic activity and lowered specific heat values of activated fluids.

The increased volatility of activated fluids as described in this article is interpreted as indicating a loosening of intermolecular hydrogen bonding [24] [25]. Thus, water is a liquid at temperatures of between $0^{\circ} \mathrm{C}-100^{\circ} \mathrm{C}$ because of its relatively strong intermolecular hydrogen bonding. Partial loosening of hydrogen bonding occurs with higher temperature but the effect is insufficient for the water vapor to match atmospheric pressure (760 mm $\mathrm{Hg}$ ) till water reaches $100^{\circ} \mathrm{C}$. Similarly, the vapor pressure of ethanol does not reach atmospheric pressure till $78.3^{\circ} \mathrm{C}$. The pressures in the closed flexible containers of ethanol that are described in this article clearly exceed atmospheric pressure, even after refrigeration.

Vapor pressure is a function of temperature and the concentration and kinetic activity of the vaporized molecules. Although not reported in this paper, the temperature of activated fluids in open containers is marginally less than the temperature in corresponding non-activated fluids. This slight reduction is a likely consequence of the increased vaporization of the activated fluids. The activated fluids also show a less concave meniscus than the corresponding control fluids; indicating a somewhat lowered surface tension. The concentrations of vapor molecules in the activated and non-activated corresponding fluids have not been determined. Still, the capacity 
of the vapor from activated fluids to readily escape from capped glass vials, even with aluminum wrapping, is very suggestive of a heighten kinetic activity of the vaporized molecules. This conclusion is consistent with the internal streaming that can be seen by sprinkling particles of neutral red dye onto activated water [24].

The persisting and continuing increased fluid vaporization caused by MgO has also been confirmed with several other fluid activating compounds. It is interpreted as evidence of continuing absorption of an environmental force. The release of the more highly kinetically activated molecules provides an explanation for the loss of activity of the lidocaine containing solution used in an earlier study on children with autism [20]. It also shows the value for maintaining activated solutions in completely sealed containers.

Other water activating compounds that have been tested include humic acid, pelleted ground and heated volcanic rock material (Kiko ${ }^{\mathrm{TM}}$ pellets), Moringa oleifera leaf extracts, lidocaine and certain other pharmaceuticals. A feature of many of these water-activating compounds is their dipolar or multipolar nature in having separated electrical charges. As discussed elsewhere, a natural force is presumably necessary to prevent the fusion and possible annihilation of opposing electrical charges [24]. This consideration led to the suggestion that electrical charges may attract a repelling natural force. Some compounds may release the absorbed force in an oscillatory manner and, thereby, be able to transfer the force to nearby fluids.

Condensed ethanol and water also show increasing activation following exposure of their vapors to the electrical energy from a Van de Graaff generator. It is likely, therefore, that once fluids are sufficiently activated they can directly absorb the environment force without the need of intermediary absorbing dipolar compounds. This is also consistent with the practice of homeopathy, in which herbal tinctures may initiate the water activation process, which can then be extended to the activation of the water used in repeated dilutions.

While significantly less that that of the test vial, the weight loss in the control vial in the year-long experiment using $\mathrm{MgO}$ was actually more than seen in many similar controls. As noted above, it is possible but not yet formally proven that activating compounds, including highly activated fluids, can mediate activation of nearby fluids even without the need for direct contact.

The proposed energy force has been termed KELEA for kinetic energy limiting electrostatic attraction. Results from various assays to be discussed elsewhere are consistent with this proposal. In the interim, it is important that the many potential benefits of using KELEA activated water in health, agriculture and industrial applications be rapidly pursued.

Clinical studies evaluating the benefits of consuming activated water in various illnesses are clearly indicated [22]. It is reasonable to equate homeopathy as a form of KELEA activated water and that its benefits are primarily achieved through the activation of the ACE pathway. Indeed, many aspects of complementary alternative medicine are readily explainable as measures for activating the ACE pathway [22].

KELEA activated water using volcanic rock pellets in a procedure referred to as Kiko ${ }^{\mathrm{TM}} \mathrm{Technology}$ has proven to provide substantial benefits in the cultivation of rice and sugarcane [26]. As noted earlier, the agricultural benefits of many commonly used components for soil amendments may well be primarily working via the activation of water. These include zeolites and humic/fulvic acids. They also include an extract called HB-101 from the sap of Japanese trees and an extract of Moringa oleifera tree leaves. Both are used in high dilutions in water and both have been shown to readily activate water as described in this article [24] [27].

The inclusion of gasoline in the present study is because of the relationship of volatility to the efficiency of combustion [28]. More rapid and complete combustion of gasoline and other liquid fuels can be expected from increased kinetic activity of hydrocarbon molecules. Indeed, preliminary data support increased mileage using KELEA activated gasoline.

Increased volatility of KELEA activated fluids is also reflected in lowered specific heat values. This has found useful application in increasing the efficiently of heat transfers in both heating and cooling operations by using KELEA activated water [29].

Finally, the physics of KELEA as a form of natural energy needs to be pursued. The weight loss assay described in this paper provides a quantitative means for the detection and possibly the shielding of KELEA. As shown in both Figure 4 and Figure 6, minor fluctuations can occur over relatively short time periods in the rate of evaporation from activated fluid samples. This could indicate periods of variable intensity of KELEA. It should also be mentioned that minor differences in the measured and relative weights of different non-fluid items have been observed in some experiments. This too needs to be explored as a possible effect of KELEA on solid objects.

In conclusion this paper presents data that an external force can, in a manner that is unrelated to temperature, 
increase the vapor pressure of liquids. The effect is most likely due to a weakening of intermolecular hydrogen bonding together with increased kinetic energy of the vapor molecules. The external force has been designated KELEA (kinetic energy limiting electrostatic attraction). KELEA can be utilized in a wide range of health, agricultural and industrial applications.

\section{Acknowledgements}

The Institute of Progressive Medicine is a component of MI Hope Inc., a public non-profit charity based in South Pasadena CA USA.

\section{References}

[1] Martin, W.J. (1992) Detection of Viral Related Sequences in CFS Patients Using the Polymerase Chain Reaction. In: Hyde, B.M., Eds., The Clinical and Scientific Basis of Myalgic Encephalomyelitis Chronic Fatigue Syndrome, Nightingdale Research Foundation Press, Ottawa, 278-283.

[2] Martin, W.J. (1994) Stealth Viruses as Neuropathogens. CAP Today, 8, 67-70.

[3] Martin, W.J., Zeng, L.C., Ahmed, K. and Roy, M. (1994) Cytomegalovirus-Related Sequences in an Atypical Cytopathic Virus Repeatedly Isolated from a Patient with the Chronic Fatigue Syndrome. American Journal of Pathology, 145, 441-452.

[4] Martin, W.J., Ahmed, K.N., Zeng, L.C., Olsen, J.-C., Seward, J.G. and Seehrai, J.S. (1995) African Green Monkey Origin of the Atypical Cytopathic "Stealth Virus" Isolated from a Patient with Chronic Fatigue Syndrome. Clinical and Diagnostic Virology, 4, 93-103. http://dx.doi.org/10.1016/0928-0197(95)00020-9

[5] Martin, W.J. (1996) Severe Stealth Virus Encephalopathy Following Chronic Fatigue Syndrome-Like Illness: Clinical and Histopathological Features. Pathobiology, 64, 1-8. http://dx.doi.org/10.1159/000163999

[6] Martin, W.J. (1995) Stealth Virus Isolated from an Autistic Child. Journal of Autism and Developmental Disorders, 25, 223-224. http://dx.doi.org/10.1007/bf02178507

[7] Martin, W.J. (1996) Simian Cytomegalovirus-Related Stealth Virus Isolated from the Cerebrospinal Fluid of a Patient with Bipolar Psychosis and Acute Encephalopathy. Pathobiology, 64, 64-66. http://dx.doi.org/10.1159/000164010

[8] Martin, W.J. and Anderson, D. (1999) Stealth Virus Epidemic in the Mohave Valley: Severe Vacuolating Encephalopathy in a Child Presenting with a Behavioral Disorder. Experimental and Molecular Pathology, 66, 19-30. http://dx.doi.org/10.1006/exmp.1999.2237

[9] Martin, W.J. and Glass, R.T. (1995) Acute Encephalopathy Induced in Cats with a Stealth Virus Isolated from a Patient with Chronic Fatigue Syndrome. Pathobiology, 63, 115-118. http://dx.doi.org/10.1159/000163942

[10] Martin, W.J (2003) Stealth Virus Culture Pigments: A Potential Source of Cellular Energy. Experimental and Molecular Pathology, 74, 210-223. http://dx.doi.org/10.1016/S0014-4800(03)00037-6

[11] Martin, W.J. (2014) Alternative Cellular Energy (ACE) Pathway in the Repair of the Cytopathic Effect (CPE) Caused by Stealth Adapted Viruses: In Vitro and in Vivo Evidence Supporting a New Therapeutic Paradigm. In: Martin, W.J., Ed., Stealth Adapted Viruses; Alternative Cellular Energy (ACE) \& KELEA Activated Water, Random House, New York, 31-70.

[12] Izaguire, R.R., Guzman, M.R., Fuentes, R.C., Mena, C.E., Penate, E., et al. (2014) Alternative Cellular Energy Based Therapy of Childhood Diarrhea. In: Martin, W.J., Ed., Stealth Adapted Viruses; Alternative Cellular Energy (ACE) \& KELEA Activated Water, Random House, New York, 103-114.

[13] Liang, S., Christner, D., Du Laux, S. and Laurent, D. (2011) Significant Neurological Improvement in Two Patients with Amyotrophic Lateral Sclerosis after 4 Weeks of Treatment with Acupuncture Injection Point Therapy Using Enercel. Journal of Acupuncture and Meridian Studies, 4, 257-261. http://dx.doi.org/10.1016/j.jams.2011.09.017

[14] Dubrov, V., Dubrova, T., Suhareva, V., Christner, D., Baiamonte, J., et al. (2012) Efficacy of Three Weeks Treatment with Enercel ${ }^{\circledR}$ for New Onset, Presumed Drug-Sensitive and Confirmed Multi-Drug Resistant Pulmonary Tuberculosis at the Regional Anti-Tuberculosis Hospital in Chernigov, Ukraine. Tuberculosis, Pulmonary Disease, HIV Infection, Vol. 1, 85.

[15] Felber, T.D., Smith, E.B., Knox, J.M., Wallis, C. and Melnick, J.L. (1973) Photodynamic Inactivation of Herpes Simplex: Report of a Clinical Trial. JAMA, 223, 289-292. http://dx.doi.org/10.1001/jama.1973.03220030027005

[16] Myers, M.G., Oxman, M.N., Clark, J.E. and Arndt, K.A. (1975) Failure of Neutral-Red Photodynamic Inactivation in Recurrent Herpes Simplex Virus Infections. The New England Journal of Medicine, 293, 945-949. http://dx.doi.org/10.1056/NEJM197511062931901

[17] Martin, W.J. and Stoneburner, J. (2005) Symptomatic Relief of Herpetic Skin Lesions Utilizing an Energy-Based Ap- 
proach to Healing. Experimental and Molecular Pathology, 78, 131-134. http://dx.doi.org/10.1016/j.yexmp.2004.10.007

[18] Martin, W.J. and Stoneburner, J. (2014) Alternative Cellular Energy (ACE) Pathway Activation as the Mode of Action of Neutral Red Dye Phototherapy of Human Viruses. Journal of Human Virology \& Retrovirology, 1, Article ID: 00019. http://dx.doi.org/10.15406/jhvrv.2014.01.00019

[19] Martin, W.J. (2014) Neutral Red Dye/Ultraviolet Light Activation of the Alternative Cellular Energy (ACE) Pathway. A Historical Overview and Current Use of Neutral Red Dye Phototherapy of Herpes Simplex Virus Infections. In: Martin, W.J., Ed., Stealth Adapted Viruses; Alternative Cellular Energy (ACE) \& KELEA Activated Water, Random House, New York, 71-86.

[20] Martin, W.J. (2014) Alternative Cellular Energy (ACE) Pathway Activation as Natural Therapy for Autism. In: Martin, W.J., Ed., Stealth Adapted Viruses; Alternative Cellular Energy (ACE) \& KELEA Activated Water, Random House, New York, 87-102.

[21] Fisher, P. (2012) What Is Homeopathy? An Introduction. Frontiers in Bioscience (Elite Edition), 4,1669-1682. http://dx.doi.org/10.2741/E489

[22] Martin, W.J. (2015) Therapeutic Potential of KELEA Activated Water. International Journal of Complementary \& Alternative Medicine, 1, Article ID: 00001.

[23] Trieschmann, R.B. (1999) Energy Medicine for Long-Term Disabilities. Disability and Rehabilitation, 21, $269-276$. http://dx.doi.org/10.1080/096382899297693

[24] Martin, W.J. (2014) KELEA Activated Water-Enhancing the Alternative Cellular Energy (ACE) Pathway. In: Martin, W.J., Ed., Stealth Adapted Viruses; Alternative Cellular Energy (ACE) \& KELEA Activated Water, Random House, New York, 115-144.

[25] Chaplin, M.F. (2010) Water’s Hydrogen Bond Strength. In: Lynden-Bell, R.M., Morris, S.C., Barrow, J.D., Finney, J.L. and Harper, C., Eds., Water and Life: The Unique Properties of $\mathrm{H}_{2} \mathrm{O}$. CRC Press, Boca Raton, 69-86. http://dx.doi.org/10.1201/EBK1439803561-c5

[26] Martin, W.J. (2015) KELEA Activated Water Leading to Improved Quantity \& Quality of Agricultural Crops. Advances in Plants \& Agriculture Research, 2, Article ID: 00033. http://dx.doi.org/10.15406/apar.2015.02.00033

[27] Martin, W.J. (2015) Do the Benefits of Moringa oleifera Trees Extend to the KELEA Activation of Water. Advances in Plants \& Agriculture Research, 2, Article ID: 00036.

[28] Martini, G., Manfredi, U., Mellios, G., Mahieu, V., Larsen, B., Farfaletti, A., Krasenbrink, A. and De Santi, G. (2007) Effects of Gasoline Vapourpressure and Ethanol Content on Evaporative Emissions from Modern Cars. In: Martini, G., Ed., Joint EUCAR/JRC/CONCAWE Study on: Effects of Gasoline Vapour Pressure and Ethanol Content on Evaporative Emissions from Modern Cars, Institute for Environment and Sustainability, European Commission DirectorateGeneral Joint Research Centre, Ispra.

[29] Martin, W.J. (2015) Improved Efficiency of Heat Exchange Using KELEA Activated Water. Open Journal of Energy Efficiency, 4, 36-43.

\author{
Abbreviation \\ ACE: Alternative Cellular Energy \\ KELEA: kinetic energy limiting electrostatic attraction \\ CPE: cytopathic effect, UV-ultraviolet \\ MgO: magnesium oxide, \\ mg: milligram \\ oz: ounce
}

